\title{
Severe Myoclonic Epilepsy in Infancy - Adult Phenotype with Bradykinesia, Hypomimia, and Perseverative Behavior: Report of Five Cases
}

\author{
P. Martin ${ }^{a}$ B. Rautenstrauß ${ }^{b}$ A. Abicht ${ }^{b} \quad$ J. Fahrbach ${ }^{a} \quad$ S. Koster ${ }^{a}$ \\ a Séguin-Clinic for Persons with Severe Intellectual Disability, Kehl-Kork, and ${ }^{\text {b}}$ Medical Genetics Center and \\ Friedrich-Baur-Institute, Munich, Germany
}

\section{Key Words}

Adult phenotype $\cdot$ Ataxia $\cdot$ Bradykinesia $\cdot$ Dravet syndrome $\cdot$ Hypomimia $\cdot$ Perseveration $\cdot$ Severe myoclonic epilepsy of infancy $\cdot$ Spasticity

\begin{abstract}
Dravet syndrome or severe myoclonic epilepsy in infancy (SMEI) is an epileptic syndrome characterised by refractory epilepsy and intellectual disability, typically presenting with febrile and afebrile generalised and unilateral clonic/ tonic-clonic seizures in the first year of life and other types of seizures appearing later in the course of the disease. Five adult patients with SMEI and SCN1A mutations are reported, in which motor and behavioural abnormalities were outstanding symptoms. Bradykinesia, responding with latency, slow speaking with a thin voice, midface hypomimia and perseveration were distinctive features in all cases. These symptoms may be fit to define the adult phenotype of SMEI beyond seizure/epilepsy criteria. The motor and behavioural symptoms are discussed in the context of a possibly underlying frontal lobe/mesofrontal and cerebellar dysfunction.

Copyright $\odot 2011$ S. Karger AG, Basel
\end{abstract}

Severe myoclonic epilepsy in infancy (SMEI) is a clinically defined entity which was described for the first time by Charlotte Dravet about 3 decades ago; it is also referred to as Dravet syndrome (DS) [Dravet et al., 1982; Engel, 2001].

SMEI is one of the severe epilepsy phenotypes associated with mainly heterozygous sequence variants or deletions of the SCN1A gene encoding the voltage-gated $\alpha$ type I sodium channel $\left(\mathrm{Na}_{\mathrm{v}} 1.1\right)$ and which is located on chromosome 2q24 [Mulley et al., 2005; Ogiwara et al., 2007]. SCN1A-related seizure disorders are inherited in an autosomal dominant manner and mutations of the SCN1A gene can be found in about $80 \%$ of cases with DS [Fujiwara, 2006; Lossin, 2009].

The incidence of SMEI is $1 / 20,000-1 / 40,000$ of the general population with a 2:1 male preponderance [Hurst, 1990; Yakoub et al., 1992; Dravet et al., 2005].

SMEI was added to the International League Against Epilepsy (ILAE) classification in 1989 where it was registered among 'epilepsies and syndromes undetermined as to whether they are focal or generalized' and is now listed under 'epileptic encephalopathies in which the epileptiform abnormalities may contribute to progressive dysfunction' [Commission on Classification and Terminology of the ILAE, 1989; Engel, 2001]. The diagnostic criteria of SMEI are listed in table 1.

\section{KARGER \\ Fax +4161306 1234 \\ E-Mail karger@karger.ch}

www.karger.com
(C) 2011 S. Karger AG, Basel

$1661-8769 / 10 / 0015-0231 \$ 26.00 / 0$

Accessible online at:

www.karger.com/msy
J. Peter Martin, MD

Séguin-Clinic for Persons with Severe Intellectual Disabilities Landstr. 1

DE-77694 Kehl-Kork (Germany)

Tel. +49 7851842 252, E-Mail pmartin@epilepsiezentrum.de 
Though most studies on DS have focussed on the epilepsy aspects of the syndrome and poorly controlled seizures remain a core problem in many if not all patients with DS, behavioural and motor features of SMEI, which have been assumed to result from a so-called epileptic encephalopathy, likewise deserve clinical and scientific recognition.

Every study on SMEI, of course, reports on the cognitive outcome of patients diagnosed with this syndrome, which is poor in almost all cases. An arrest or deterioration of cognitive development in previously normal children is recognised between 1 and 4 years of age, starting in the second year of life in most cases and leading to moderate or severe intellectual disability (ID) [Dravet et al., 2005; Wolff et al., 2006; Ragona et al., 2010]. In few patients, only a more favourable cognitive outcome with only mild ID has been reported [Incorpora, 2009; Ragona et al., 2010].

Neurological signs and symptoms in SMEI are mentioned in the literature on the sidelines only. Authors most frequently refer to cerebellar and pyramidal signs and tremor, but dystonia, dyskinesia and bradykinesiarigidity as well as hemiparesis were also reported [Dravet et al., 1982, 2002, 2005; Yacoub et al., 1992; Oguni et al., 2001; Jansen et al., 2006; Ragona et al., 2010].

There are only few data on neuropsychological and behavioural findings in individuals with DS. In a study on neuropsychological aspects in 20 children with DS, poor visuomotor skills were found in all children aged 4-13 years together with behavioural traits of hyperactivity, poor relational capacities and gestural stereotypies [Wolff et al., 2006]. Hyperactivity and attention deficits are reported in other studies on children with DS [Yakoub et al., 1992; Dravet et al., 2002; Ragona et al., 2010]. In adults with SMEI, however, a behavioural change seems to become obvious and 'behaviour is no more hyperactive and rather characterised by slowness and perseverance' [Dravet et al., 2002].

Here, 5 cases of adults with SMEI and SCN1A mutations are reported, showing a common distinctive motorbehavioural phenotype with bradykinesia, hypomimia, bradyphrenia and perseverative utterances, besides ataxia and spasticity. The first patient was diagnosed with DS according to epilepsy criteria (and in contempt of his severe motor impairment) years before mutations of the SCN1A gene could be detected in DS. However, once the SCN1A mutation had been detected in this patient, years after the initial clinical diagnosis of SMEI, the authors realized the DS-signalling motor and behavioural symptoms in the subsequent cases.
Table 1. Diagnostic criteria of severe myoclonic epilepsy in infancy

Family history of epilepsy or febrile convulsions

Normal development before epilepsy onset

Seizures beginning during the first year of life in the form of generalised or unilateral febrile clonic seizures

Secondary appearance of myoclonic jerks, and often partial seizures

EEGs show generalised spike-waves and polyspike-waves, early photosensitivity, and focal abnormalities

Retardation of psychomotor development from the second year of life on

Ataxia, pyramidal signs, and interictal myoclonus appear in the course of the disease

Resistance to all forms of treatment

Commission on Classification and Terminology of the ILAE, 1989.

\section{Case Reports}

\section{Patient A}

This 44-year-old male patient was born to healthy nonconsanguineous parents without complications (vaginal delivery) after an uneventful pregnancy. The patient's birth weight was 3,300 g, length $54 \mathrm{~cm}$ and occipitofrontal circumference $36 \mathrm{~cm}$; Apgar score was 10 at $5 \mathrm{~min}$. There was no family history of epilepsy or febrile seizures, ID or motor disorders. His psychomotor development seemed to be normal until the age of 8 months, when he started to speak single words. A few months later he stopped speaking. He again engaged in communication at the age of 4 years using meaningful words and short sentences; he started to walk unaided, but his movements were slow and gait ataxia was soon described. From the beginning of his fourth decade on he developed a slowly progressive spasticity of his legs and was only able to walk 1-2 m independently.

$\mathrm{He}$ attended a special school for children with learning disabilities and could not acquire basic arithmetic and reading skills. After school, he worked in a centre for sheltered work and lived in a residential home.

Epilepsy in patient A started with a febrile generalised tonicclonic seizure (GTCS) a few days after birth, and he experienced another 6 fever associated GTCS during his first year. In spite of an anticonvulsive treatment with phenobarbitone and primidone, the course of his epilepsy was unfavourable with several GTCS/ month. Tonic seizures, atypical absences, obtundation status, and complex-partial seizures emerged over time. He experienced a generalised convulsive status epilepticus when he was 5 and 15 years old. Despite of numerous changes of the anticonvulsive treatment, the epilepsy has not been sufficiently controlled with 2 or 3 GTCS occurring per week. Since the patient was 35 years old, recurrent hallucinatory symptoms - he was convinced that a dog is around and threatens him - appeared.

On neuropsychiatric evaluation at 44 years, the patient spoke very slowly with a thin, dysarthrophonic voice. He used sentenc- 
es with 3 to 5 words, responding with substantial latency and with a significant perseverative reiteration. His mood was balanced with small modulation. No indication of delusional or hallucinatory symptoms was present. Neurological examination revealed a fragmentation of smooth pursuit movements of the eyes, a horizontal gaze-dependent nystagm to both sides, marked hypomimia of the midface, and drooling saliva. There was a marked spasticity more pronounced in the legs than in the arms and no evidence of sensory dysfunctions. Deep tendon reflexes of the 4 limbs were brisk, more pronounced in the legs but without a Babinski's sign. There was mild dysmetria in the finger to nose test and asynergy in rapid-alternating supination-pronation tests on both sides as well as an upper-limb tremor during action. All movements were markedly slowed. Stance and gait showed postural instability and a spastic-ataxic gait disturbance was obvious and pronounced genua valga.

The EEG at 44 years showed a slowed background slowing (6-7 Hz) but no focal slowing or epileptiform activity.

The cerebral magnetic resonance imaging (MRI) at 37 years revealed a reduced volume of the forebrain and mild cerebellar atrophy. There was slightly increased signal and reduced volume of the periventricular white matter, spreading into the subcortical white matter of the left inferior frontal gyrus.

Sequence analysis identified a novel SCN1A mutation in exon 14 (c.2473_2476delTACT; p.Tyr825fs; exon 14).

\section{Patient $C$}

This 19-year-old male patient was born to healthy, nonconsanguineous parents. Pregnancy and delivery were uneventful (36 weeks gestation, body weight 2,140 g, Apgar scores 9 and 10 at 1 and $5 \mathrm{~min}$, respectively). Respiratory problems during the neonatal period were reported. There was no family history of neuropsychiatric disorders, especially not of epileptic seizures or febrile convulsions. Early development was normal until the age of 3 years, except for a mildly delayed language acquisition. Thereafter, a decline in cognitive-intellectual functions was recognised leading to severe learning disabilities. He was able to speak 4-word sentences at 3 years but did not gain more language abilities; his speech became more and more slurred. Motor functions developed almost normal until about 5 years (he was able to run, jump and swim) but declined thereafter, in parallel with an increase in seizure activity. At 14 years of age, a scoliosis was operated on; from then on, the patient experienced a slow loss of motor functions, increasing rapidly during the last years.

First seizures were witnessed in the patient when he was 11 months old: staring spells with a version of the head. Shortly afterwards, a febrile GTCS occurred and, another 6 months later, myoclonic seizures appeared together with lateralised GTCS and atypical absence seizures/obtundation status; temporarily convulsive seizures occurred preferentially during sleep. Nearly all classical and newer anticonvulsive drugs (including stiripentol) as well as vagus nerve stimulation therapy failed to achieve seizure control.

Neuropsychiatric examination at 19 years revealed significantly delayed response time; the patient answered correctly to questions with a lag up to $60 \mathrm{~s}$. His speech was slurred and slowed with a thin voice. He was able to talk in short incomplete sentences which he reiterated in a perseverative manner. A fragmentation of smooth pursuit movements of the eyes was noticeable but no abnormal nystagm. There was a marked hypomimia of the patient's midface and drooling with wide-opened mouth. He was able to protrude his tongue but failed to move it sidewards. There were no sensory deficits detectable but a significant spasticity of the trunk and limbs, pronounced on legs and on the left side. Deep tendon reflexes of the arms were normal (slight accentuation on the right side), but there was a symmetric hyperreflexia in the lower limbs without extensor plantar response. Disequilibrium with a very slow spastic-ataxic gait was noted and marked genua valga.

EEG revealed bilateral $\tau$-activity $(5-6 / s)$ over the frontal regions with superimposed slow $\tau-\delta$ waves and bifrontal sharp waves.

MRI at 16 years showed a widening of the subarachnoid space over both frontal lobes and of the lateral ventricles as well as a mild atrophy of the cerebellum.

Sequence analysis identified an already known missense SCN1A mutation in exon 16 (c.2947-G>T; p.Val983Phe) [Kanai et al., 2004].

Developmental history, epilepsy data, clinical, MRI, and EEG as well as molecular genetic findings of 2 additional female (patient $\mathrm{D}, \mathrm{E}$ ) and 1 male (patient $\mathrm{B}$ ) patients have much in common with the cases reported above and are listed together with the latter in tables 2-6. Photos of each patient are shown in figure 1 . The epilepsy therapy proved to be difficult in all patients; almost all anticonvulsive drugs, including bromide, were employed as treatment.

\section{Discussion}

DS is primarily recognised and defined as an epileptic syndrome in infancy and childhood. There are no more detailed reports on motor functions and behaviour characteristics of patients diagnosed with this syndrome in adulthood. Even a recently published long-term follow-up study of DS up to adulthood mentioned only 'ataxic gait' in 13 of 31 adult patients enclosed in the study and 'clumsiness' in 11 subjects; 4 patients were reported to have experienced 'gradual deterioration in motor ability' during adolescence and/or adulthood [Akiyama et al., 2010].

A few years ago, we saw a patient (patient A) with considerable motor impairment and a characteristic epilepsy history suggestive of SMEI. Because of the markedness of his movement disorder, we initially hesitated to diagnose him with DS but initiated genetic analysis as soon as it became routinely disposable. Thereafter, we recognised further SMEI patients with very similar motor and behavioural characteristics, the history and clinical/paraclinical findings of which are presented above.

Spasticity and ataxia in some of the patients were so pronounced that they had been diagnosed with cerebral palsy previously. Especially the history of gestational bleeding during the last trimester in 1 patient (patient $\mathrm{D}$ ) as well as placental insufficiency and caesarean delivery reported in another (patient $\mathrm{E}$ ) suggested this diagnosis. 
Table 2. Developmental history of 5 patients with SMEI

\begin{tabular}{|c|c|c|c|c|c|}
\hline & Patient A & Patient B & Patient C & Patient D & Patient E \\
\hline Sex & male & male & male & female & female \\
\hline Age & 44 years & 28 years & 19 years & 33 years & 32 years \\
\hline Pregnancy & uneventful & uneventful & uneventful & gestational bleeding at 7 th month & uneventful \\
\hline Delivery & uneventful & 42nd week, uneventful & uneventful & uneventful & caesarean delivery \\
\hline First words & 8 months & 18 months & slightly delayed & 24 months & delayed \\
\hline Unaided sitting & 6 months & 6 months & 6 months & 6 months & 2nd year \\
\hline Unaided walking & 4 years & 6 months & 1 year & 14 months & 2.5 years \\
\hline Rapid intellectual decline* & end 1 st/beginning 2 nd year & no & 3 years & no & no \\
\hline Rapid motor decline* & end 1 st/beginning 2 nd year & no & 3 years & no & no \\
\hline Slow intellectual decline ${ }^{* *}$ & not obvious & no & questionable & no & no \\
\hline Slow motor decline $e^{* *}$ & during adulthood & questionable & from 15 years on & from about 20 years on & from about 15 years on \\
\hline Arithmetic and reading skills & no & no & no & to some extent & no \\
\hline Hyperactivity & no & no & no & during childhood, until 13 years & toddler stage \\
\hline
\end{tabular}

* Over less than 1 year. ${ }^{* *}$ Over more than 1 year.

Table 3. Epilepsy data of 5 patients with SMEI

\begin{tabular}{|c|c|c|c|c|c|}
\hline & Patient A & Patient B & Patient $\mathrm{C}$ & Patient D & Patient E \\
\hline Family history of febrile seizures & no & no & no & no & no \\
\hline Family history of epilepsy & no & no & no & no & no \\
\hline First seizure/epilepsy onset & 1 week & 9 months & 11 months & 2 months & 7 months \\
\hline Febrile seizures & yes & yes & yes & yes & yes \\
\hline Seizures triggered by warm environment & no & no & no & no & no \\
\hline Other seizure-provoking factors & not known & emotional stress & not known & $\begin{array}{l}\text { watching movies, } \\
\text { doing a puzzle }\end{array}$ & no \\
\hline$\overline{\text { GTCS }}$ & yes & & yes & yes & yes \\
\hline Hemiconvulsive seizures & no & yes & yes & yes & yes \\
\hline Frequent nocturnal seizures & no & yes & yes & no & yes \\
\hline Convulsive status epilepticus & yes & no & no & yes & no \\
\hline Maximal GTCS frequency & several/month & 30/month & 20/month & 24/month & $10 /$ month \\
\hline Myoclonic seizures & no & yes & yes & yes & questionable \\
\hline Absence seizures & yes & yes & yes & no & yes \\
\hline Obtundation status & yes & yes & yes & no & yes \\
\hline Tonic seizures & yes & yes & no & yes & no \\
\hline Complex partial seizures & yes & yes & no & yes & no \\
\hline Other focal seizures & no & no & no & yes & yes \\
\hline Seizure freedom more than 6 months & no & no & yes & no & $\begin{array}{l}\text { yes ( } 12 \text { years } \\
\text { under bromide) }\end{array}$ \\
\hline Therapy-resistant epilepsy & yes & yes & yes & yes & yes \\
\hline
\end{tabular}

In adults, it was not easy to trace back a rapid motor and/or intellectual decline during infancy/early childhood. The history, however, revealed a slow and steadily decreasing motor ability in at least 4 of the 5 patients presented. This raised the question of whether a defined neurodegenerative or neurometabolic disease was the underlying disorder.

Mol Syndromol 2010;1:231-238
The progressive development of motor symptoms in patients with DS possibly has its counterpart in findings from animal studies that revealed an upregulation of $\mathrm{Na}_{\mathrm{v}}$ 1.1 expression in normally developing mice during the first postnatal weeks but not in mice carrying an SCN1A mutation [Ogiwara et al., 2007].

Hypomimia in all of our patients, together with bradykinesia and abnormal latency in initiating movements 
Table 4. Clinical findings in 5 patients with SMEI

\begin{tabular}{|c|c|c|c|c|c|}
\hline & Patient A & Patient B & Patient C & Patient D & Patient E \\
\hline Intellectual disability & severe & moderate & moderate & moderate & moderate \\
\hline Psychiatric disorders & $\begin{array}{l}\text { episodes with visual } \\
\text { hallucinations/delusions }\end{array}$ & no & no & no & no \\
\hline Responding with latency & yes (marked) & yes (slightly) & yes (marked) & yes (slightly) & yes \\
\hline Slow speaking & yes (marked) & yes & yes (marked) & yes & yes (marked) \\
\hline Verbal perseveration & yes (marked) & yes & yes (marked) & yes & yes (marked) \\
\hline Dysarthrophonia & yes & yes & yes & yes & yes \\
\hline Thin voice & yes & yes (mild) & yes & yes & yes \\
\hline $\begin{array}{l}\text { Disturbed eye } \\
\text { movements }\end{array}$ & $\begin{array}{l}\text { fragmentation of } \\
\text { smooth pursuit, hori- } \\
\text { zontal gaze nystagm }\end{array}$ & no & $\begin{array}{l}\text { fragmentation of } \\
\text { smooth pursuit }\end{array}$ & no & no \\
\hline Drooling saliva & yes & no & yes & no & no \\
\hline Midface hypomimia & yes & yes & yes & yes & yes \\
\hline Bradykinesia & yes & yes & yes & yes & yes \\
\hline Spasticity & yes & questionable & $\begin{array}{l}\text { yes, more pronounced } \\
\text { in the legs and on the } \\
\text { left side }\end{array}$ & yes, in the legs, mild & $\begin{array}{l}\text { yes, more pronounced } \\
\text { in the legs and on the } \\
\text { right side }\end{array}$ \\
\hline Rigor & no & no & no & no & no \\
\hline Ataxia & yes & yes & yes & yes & yes \\
\hline Spasticity and ataxia & yes & questionable & yes & yes & yes \\
\hline Ability to walk & aided & aided & aided & unaided & aided \\
\hline Tremor & $\begin{array}{l}\text { action tremor, } \\
8-10 \mathrm{cp} / \mathrm{s}\end{array}$ & $\begin{array}{l}\text { with action and } \\
\text { sustained position, } \\
8-10 \mathrm{cp} / \mathrm{s}\end{array}$ & $\begin{array}{l}\text { mild with action and } \\
\text { sustained position, } \\
8-10 \mathrm{cp} / \mathrm{s}\end{array}$ & $\begin{array}{l}\text { with action and } \\
\text { sustained position, } \\
8-10 \mathrm{cp} / \mathrm{s}\end{array}$ & $\begin{array}{l}\text { action tremor } \\
8-10 \mathrm{cp} / \mathrm{s}\end{array}$ \\
\hline
\end{tabular}

Table 5. MRI and EEG findings in 5 patients with SMEI

\begin{tabular}{|c|c|c|c|c|c|}
\hline & Patient A & Patient B & Patient C & Patient D & Patient E \\
\hline EEG & at 44 years & at 25 years & at 19 years & at 34 years & at 27 years \\
\hline Background activity & $6-7 / \mathrm{s}$ & $6 / s$ & $5-6 / s$ & irregular, $\alpha-\beta-\tau$ & irregular, $\alpha-\beta-\tau$ \\
\hline Focal slowing & no & no & bifrontal $\tau$ and $\delta$ & no & right frontal $\tau$ \\
\hline Focal epileptiform activity & no & bifrontal sharp waves & bifrontal sharp waves & no & no \\
\hline $\begin{array}{l}\text { Generalised/bisynchronous } \\
\text { epileptiform activity }\end{array}$ & no & no & no & no & no \\
\hline MRI & at 37 years & at 25 years & at 16 years & not done & not done \\
\hline Supratentorial atrophy & bifrontal atrophy (hypoplasia) & bifrontal atrophy & bifrontal atrophy & - & - \\
\hline Infratentorial atrophy & mild cerebellar atrophy & no & mild cerebellar atrophy & - & - \\
\hline White matter lesions & $\begin{array}{l}\text { periventricular white matter slightly } \\
\text { reduced and hyperintense (T2) } \\
\text { reaching to the left inferior frontal gyrus }\end{array}$ & no & no & - & - \\
\hline Cortical lesions & no & no & no & - & - \\
\hline Lesions of the basal ganglia & no & no & no & - & - \\
\hline Other lesions & no & no & no & - & - \\
\hline
\end{tabular}

would not have been typical findings in cerebral palsy. These symptoms resembled those seen in Parkinson's disease, but there could be no rigor found in patients with DS; the tremor they showed was of higher frequency than parkinsonian tremor and was a tremor at sustained posi- tion and action tremor. Moreover, the hypomimia of our patients was accentuated in the midface.

Ataxia was present in all of our patients. Four of 5 SMEI patients showed additional clear-cut spasticity of different severity, which was pronounced in the lower 
Table 6. Molecular genetic findings in 5 patients with SMEI

\begin{tabular}{lll}
\hline Patient & $\begin{array}{l}\text { SCN1A deletion } \\
\text { or duplication }\end{array}$ & SCN1A mutation \\
\hline A & no & c.2473_2476delTACT, p.Tyr825fs; exon 14; novel \\
B & no & c. 1034 G $>$ A, p.Cys345Tyr; Depienne et al., 2009 \\
C & no & c.2947-g>T, Val983Phe; exon 16; Kanai et al., 2004 \\
D & no & c.760_763delACTG, p.Thr254CysfsX4; novel \\
E & no & c. $3705+5 G>$ A; Ebach et al., 2005 \\
\hline
\end{tabular}
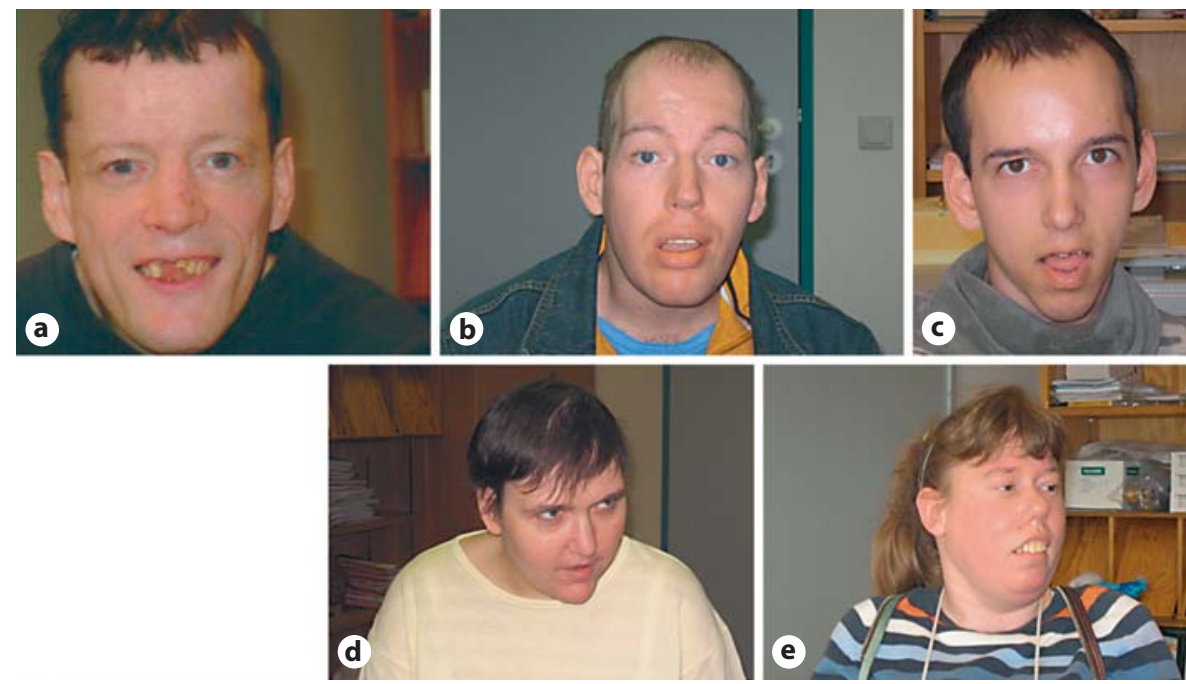

Fig. 1. Hypomimia in adult patients with Dravet syndrome: patients A-E (a-e). limbs. The motor impairment prevented all but one patient from walking independently.

MRI of the brain was available in 3 patients only. None of these had marked cerebellar atrophy or other pathology of the cerebellum. In all 3 patients, a bilateral atrophy of the frontal lobes was obvious. Gait disorders, as well as dysathria, recognised in our patients could be well explained in terms of a frontal lobe disorder. Our findings, however, are not at all sufficient to justify the diagnosis of frontal ataxia. The oculomotor signs in 2 patients (A, C) likewise speak in favour of a, at least additional, cerebellar dysfunction in these individuals. In this context, data from animal models of human SCN1A syndromes are to be considered. In a knockout mouse model of DS, homozygous mutants exhibited ataxia and seizures beginning 9 days after birth, leading progressively to mild limb tremors, side-to-side swaying and complete loss of postural control; mice genetically engineered to harbour a pathogenetic SMEI mutation (R1407X) in the SCN1A gene likewise developed an unstable gait, besides tonic- clonic and clonic seizures [Yu et al., 2006; Ogiwara et al., 2007]. Possibly corresponding to these findings, cerebellar Purkinje neurons from $\mathrm{Na}_{\mathrm{v}}$ mutant mice demonstrated significantly decreased sodium currents, which may serve as an explanation for the ataxia in these animals [Kalume et al., 2007].

Nevertheless, concomitant motor (other than ataxia) and behavioural symptoms in our patients point to the frontal lobes being involved in the pathomechanism of the phenotype we saw consistently: bradykinesia, responding with latency, slow speaking with a thin voice, midface hypomimia and verbal perseveration. These clinical symptoms match in parts (our patients with DS were probably more energetic and tenacious in their perseverative behaviour) with the behavioural complex described by Fisher [1983] and termed 'bradyphrenia' or 'abulia minor' by the author himself. Lack of spontaneity and initiative, slowness and poverty of movement, mental slowness and apathy, verbal and motor response given after a delay of up to several minutes, faint voice as well 
as perseveration were mentioned by this author among the difficulties experienced by bradyphrenics. He related these clinical features anatomically to the frontal lobes and biochemically to the dopamine (DA) system; i.e. a dysfunction of the dopaminergic afferents from the midbrain monaminergic nuclei (ventral tegmental area) to the frontal lobes, the s.c. mesofrontal (-cortical) system [Fisher, 1983; Gaspar et al., 1992]. Fisher [1983] discussed whether abulia resulted from a defect in the same system that is excessively activated in behavioural hyperactivity. This could be of significance with respect to the observation that many patients with DS are hyperactive during childhood and get slow and perseverative in adolescence or adulthood [Dravet et al., 2002]. In 2 of our patients, the history revealed unequivocally hyperactive behaviour during infancy and childhood, changing thereafter to the phenotype described above. There is much evidence for assuming that DA plays a crucial role in cortical regulation of motor and cognitive processes, and that a dysregulation of frontal dopaminergic neurotransmission is likely to contribute to attention-deficit/hyperactivity disorder [Heijtz et al., 2007]. However, at the cellular and synaptic level, the effects of DA in the frontal cortex are highly complex and opposing with respect to different receptor subtypes (D1- vs. D2-like) and are likely to display different age-dependent effects in normal developing as well as in early brain damage [Heijtz et al., 2007; Durstewitz and Seamans, 2008; Boyce and Finlay, 2009].

The striking hypomimia in our patients falls into line with the assumption of a frontal dysfunction, as facial movements are under powerful influence of the frontal cortex and emotional facial expressions are known to be impoverished in frontal cerebral pathology [Weddell et al., 1990; Morecraft et al., 2004; Juckel et al., 2010].

Studies on the spatial distribution of $S C N 1 A / \mathrm{Na}_{\mathrm{v}} 1.1$ expression in rodent and human brain are in accordance with the assumption of a frontal/mesofrontal and additional cerebellar dysfunction underlying the DS motorbehavioural phenotype. In a report on the distribution of voltage-gated sodium channel $\alpha$ subunit proteins in post-mortem adult human brain, sodium-channel type I could be localised to the cerebral cortex (middle frontal gyrus, middle temporal gyrus, visual cortex, and sensory-motor cortex) as well as to the cerebellar cortex [Whitaker et al., 2001]. In mice, $\mathrm{Na}_{\mathrm{v}} 1.1$ expression could be observed in neocortical interneurons, the hippocampus, the cerebellum Purkinje cells, and deep cerebellar nuclei but also in the thalamus and various brainstem regions, distinctively the ventral tegmental area, and also in the spinal cord [Ogiwara et al., 2007; Duflocq et al., 2008].

It does not seem to be likely that the severity of the previously described movement and behavioural disorders in DS depends on the number or frequency of seizures the patients had experienced; one of our patients (patient E) showed large-scale bradykinesia, hypomimia and perseveration but was seizure free for 12 years under bromide. The possible relation between the development of the motor symptoms and anticonvulsive drugs cannot be analysed sufficiently in the group of patients described in this report as all patients had been treated with almost every drug available. Children and adults with DS in our and other German centres receive bromide very often and over long periods of time; therefore, this compound should deserve particular consideration. There are, however, no reports on motor symptoms appearing under long-term treatment with bromide. All but one of our patients were on valproic acid (VPA) at their most recent examination. In single cases, VPA can induce a chronic encephalopathy with parkinsonian symptoms and ataxia in some of the affected individuals, but these symptoms are reversible after VPA withdrawal [Armon et al., 1996]. Moreover, our patient who had not received VPA for years (patient C) showed most pronounced bradykinesia, hypomimia and gait disturbance when he was examined.

To work out the motor-behavioural phenotype of SMEI more precisely, and to further clarify questions of causality, our group has prepared a comparative study with elaborated instruments and larger groups of DSand non-DS patients with difficult-to-treat epilepsies and ID.

References

Akiyama M, Kobayashi K, Yoshinaga H, Ohtsuka Y: A long-term follow-up study of Dravet syndrome up to adulthood. Epilepsia 51: 1043-1052 (2010).

-Armon C, Shin C, Miller P, Carwile S, Brown E, et al: Reversible parkinsonism and cognitive impairment with chronic valproate use. Neurology 47:626-635 (1996).

Boyce PJ, Finlay JM: Extracellular dopamine and norepinephrine in the developing rat prefrontal cortex: transient effects of early partial loss of dopamine. Brain Res Bull 79:104110 (2009).

-Commission on Classification and Terminology of the International League against Epilepsy: Proposal for revised classification of epilepsies and epileptic syndromes. Epilepsia 30: 389-399 (1989). 
Depienne C, Trouillard O, Saint-Martin C, An I, Bouteiller D, et al: Spectrum of SCN1A gene mutations associated with Dravet syndrome: analysis of 333 patients. J Med Genet 46:183191 (2009).

Dravet C, Roger J, Bureau M, Dalla Bernardina B: Myoclonic epilepsies in childhood, in Akimoto H (ed): Advances in Epileptology, pp 135-140 (Raven Press, New York, 1982).

Dravet C, Bureau M, Oguni H, Fukuyama Y, Cokar O: Severe myoclonic epilepsy in infancy (Dravet syndrome), in Roger J, Bureau M, Dravet C, Genton P, Tassinari CA, Wolf P (eds): Epileptic Syndromes in Infancy, Childhood and Adolescence, pp 81-103 (John Libbey, Eastleigh 2002).

Dravet C, Bureau M, Oguni H, Fukuyama Y, Cokar O: Severe myoclonic epilepsy in infancy: Dravet syndrome. Adv Neurol 95:71-102 (2005).

Duflocq A, Le Bras B, Bullier E, Couraud F, Davenne $\mathrm{M}: \mathrm{Na}_{\mathrm{v}} 1.1$ is predominantly expressed in nodes of Ranvier and axon initial segments. Mol Cell Neurosci 39:180-192 (2008).

Durstewitz D, Seamans JK: The dual-state theory of prefrontal cortex dopamine function with relevance to catechol-omethyltransferase genotypes in schizophrenia. Biol Psychiatry 64:739-749 (2008).

Ebach K, Joos H, Doose H, Stephani U, Kurlemann G, et al: SCN1A mutation analysis in myoclonic astatic epilepsy and severe idiopathic generalized epilepsy of infancy with generalized tonic-clonic seizures. Neuropediatrics 36:210-213 (2005).

Engel J: A proposed diagnostic scheme for people with epileptic seizures and with epilepsy: report of the ILAE task force on classification and terminology. Epilepsia 42:796-803 (2001).

-Fisher CM: Honored guest presentation: abulia minor vs. agitated behaviour. Clin Neurosurg 31:9-31 (1983).
Fujiwara T: Clinical spectrum of mutations in SCN1A gene: severe myoclonic epilepsy in infancy and related epilepsies. Epilepsy Res 70 Suppl 1:S223-S230 (2006).

-Gaspar P, Stepniewska I, Kaas JK: Topography and collateralization of the dopaminergic projections to motor and lateral prefrontal cortex in owl monkeys. J Comp Neurol 325: 1-21 (1992).

Heijtz RD, Kolb B, Forssberg H: Motor inhibitory role of dopamine D1 eceptors: implications for ADHD. Physiol Behav 92:155-160 (2007)

Hurst DL: Epidemiology of severe myoclonic epilepsy of infancy. Epilepsia 31:397-400 (1990).

Incorpora G: Dravet syndrome. It J Ped 35:27 (2009).

Jansen FE, Sadleir LG, Harkin LA, Vadlamudi L, McMahon JM, et al: Severe myoclonic epilepsy of infancy (Dravet syndrome): recognition and diagnosis in adults. Neurology 67: 2224-2226 (2006).

Juckel G, Mergl R, Brüne M, Villeneuve I, Frodl $\mathrm{HJ}$, et al: Is evaluation of humorous stimuli associated with frontal cortex morphology? A pilot study using facial micro-movement analysis and MRI. Cortex 2010, E-pub ahead of print.

Kalume F, Yu FH, Westenbroek RE, Wcheuer T, Catterall WA: Reduced sodium current in Purkinje neurons from $\mathrm{Na}_{\mathrm{v}} 1,1$ mutant mice: implications for ataxia in severe myoclonic epilepsy in infancy. J Neurosci 27:1106511074 (2007).

Kanai K, Hirose S, Oguni H, Fukuma G, Shirasaka Y, et al: Effect of localization of missense mutations in SCN1A on epilepsy phenotype severity. Neurology 63:329-334 (2004).

Lossin C: A catalog of SCN1A variants. Brain Dev 31:114-130 (2009).

Morecraft RJ, Stilwell-Morecraft RS, Rossing WR: The motor cortex and facial expression: new insights from neuroscience. Neurologist 10:235-249 (2004).
- Mulley JC, Scheffer IE, Petrou S, Dibbens LM, Berkovic SF, et al: SCN1A mutations in epilepsy. Hum Mutat 25:535-542 (2005).

- Ogiwara I, Miyamoto H, Morita N, Atapour N, Mazaki E, et al: $\mathrm{Na}_{\mathrm{v}} 1.1$ lokalizes to axons of Parvalbumin-positive inhibitory interneurons: a circuit basis for epileptic seizures in mice carrying an Scnla gene mutation. J Neurosci 27:5903-5914 (2007).

- Oguni H, Hayashi K, Awaya Y, Fukuyama Y, Osawa M: Severe myoclonic epilepsy in infants - a review based on the Tokyo Women's Medical University series of 84 cases. Brain Dev 23:736-748 (2001).

Ragona F, Brazzo D, De Giorgi I, Morbi M, Freri E, et al: Dravet syndrome: early clinical manifestations and cognitive outcome in 37 Italian patients. Brain Dev 32:71-77 (2010).

SCN1A variant database. http://www.molgen. ua.ac.be/SCN1AMutations/Default.cfm.

-Weddell RA, Miller JD, Trevarthen C: Voluntary emotional facial expressions in patients with cerebral lesions. Neuropsychologia 28:49-60 (1990).

-Whitaker WRJ, Faull RLM, Waldvogel HJ Plumpton CJ, Emson PC, Clare JJ: Comparative distribution of voltage-gated sodium channel proteins in human brain. Mol Brain Res 88:37-53 (2001)

Wolff M, Cassé-Perrot C, Dravet C: Severe myoclonic epilepsy of infants (Dravet syndrome): natural history and neuropsychological findings. Epilepsia 47 Suppl 2:45-48 (2006).

- Yakoub M, Dulac O, Jambaqué I, Chiron C, Poulin P: Early diagnosis of severe myoclonic epilepsy in infancy. Brain Dev 14:299-303 (1992).

- Yu FH, Mantegazza M, Westenbroek RE, Robbins CA, Kalume F, et al: Reduced sodium current in GABAergic interneurons in a mouse model of severe myoclonic epilepsy in infancy. Nat Neurosci 9:1142-1149 (2006). 